\title{
Rare presentation of small bowel adenocarcinoma with neuroendocrine differentiation in the jejunum: A case report and summary of diagnostic and management options
}

\author{
EMANUELA CIMPEANU ${ }^{1}$, WAHIB ZAFAR ${ }^{2}$, IOANA CIRCIUMARU $^{3}$, \\ ARIEL PROZUMENSHIKOV $^{1}$ and SHAMIM SALMAN ${ }^{4}$ \\ ${ }^{1}$ Department of Internal Medicine, Richmond University Medical Center, Staten Island, NY 10310; \\ ${ }^{2}$ Department of Hematology and Medical Oncology, Westchester Medical Center, Valhalla, NY 10595;

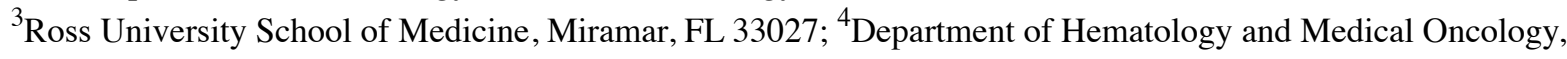 \\ Richmond University Medical Center, Staten Island, NY 10310, USA
}

Received March 18, 2019; Accepted August 30, 2019

DOI: $10.3892 / \mathrm{mco} .2019 .1925$

\begin{abstract}
Cancer of the small bowel (SB) is rare. The most common SB malignancy is neuroendocrine, followed by adenocarcinoma. SB adenocarcinoma with overlapping neuroendocrine differentiation (NED) is very uncommon. The present case report discusses the case of a young African American female who presented with high-grade SB perforation and underwent urgent surgical repair. Histology of the tumor revealed jejunal adenocarcinoma with NED. To the best of our knowledge, this type of tumor has not yet been reported in the jejunum. Referring patients that present with insidious and nonspecific abdominal discomfort for imaging studies that are more sensitive to the SB could potentially identify tumors earlier, prevent the development of emergent gastrointestinal complications and offer a chance for cure. An overview of diagnostic tools for SB evaluation was provided.
\end{abstract}

\section{Introduction}

Small bowel (SB) cancers are rare entities, accounting for only about $2 \%$ of gastrointestinal (GI) malignancies in the United States (U.S.) (1). Its incidence in the U.S. has almost doubled between 1973 and 2004 (2). Early diagnosis poses a challenge, as presenting symptoms, usually insidious and nonspecific abdominal discomfort, are often-times vague and difficult to recognize, which leads to an average delay in diagnosis of 6-12 months $(3,4)$. Less than $25 \%$ of tumors are discovered at stage 1 or 2 (3). Diagnosis is usually made when the patient

Correspondence to: Dr Emanuela Cimpeanu, Department of Internal Medicine, Richmond University Medical Center, 355 Bard Avenue, Staten Island, NY 10310, USA

E-mail: emma_ver@yahoo.com

Key words: small bowel cancer, adenocarcinoma, neuroendocrine differentiation, jejunum, chemotherapy, imaging studies has an acute GI emergency such as SB obstruction (40\%) or GI bleed (24\%) (4). SB cancer is difficult to diagnose, frequently presents at advanced stages, and has poor prognosis $(3,4)$. While surgical resection is the treatment of choice for localized tumors, no standard treatment protocol has been established for unresectable or metastatic disease (4). We present the case of a young woman with a case of infiltrating jejunal adenocarcinoma with neuroendocrine differentiation (NED), whose diagnosis was made when she came in with SB perforation.

\section{Case report}

A 45-year-old African American woman with a past medical history of deep venous thrombosis, morbid obesity (body mass index of 44), anemia, cholelithiasis, chronic back pain, gastritis, metromenorrhagia and depression presented with severe abdominal pain, nausea, vomiting, diarrhea, occasional flushing of the skin, malaise, fever, shortness of breath, a recent 20-pound weight loss and no urine output for two days. Vital signs were: Blood pressure 74/49 $\mathrm{mmHg}$, heart rate $140 \mathrm{bp}$, respiratory rate 22 bpm, temperature 100.9 F. Laboratory workup included white blood count $29.9 \mathrm{k} / \mu \mathrm{l}$, red blood count $3.37 \mathrm{~m} / \mu \mathrm{l}$, hemoglobin $7.5 \mathrm{~g} / \mathrm{dl}$, platelet count $925 \mathrm{k} / \mu \mathrm{l}$, blood urea nitrogen $36 \mathrm{mg} / \mathrm{dl}$ and creatinine $4.8 \mathrm{mg} / \mathrm{dl}$. Physical examination demonstrated generalized abdominal tenderness and guarding, left flank pain and a 10 by $10 \mathrm{~cm}$ epigastric mass. Computed tomography (CT) of abdomen was significant for a necrotic mass in the jejunum and high-grade SB perforation. Urgent surgery revealed a necrotic, perforated jejunal tumor invading the transverse colon. Histologically, the malignancy was classified as infiltrating adenocarcinoma with areas overexpressing synaptophysin, consistent with neuroendocrine differentiation. In addition, solid areas with mucin production and Signet-ring cells were also identified (Figs. 1 and 2). Immunohistochemically, the tumor cells were positive for CDX2, CK20, and patchy positive for synaptophysin. CK7, PAX-8, CD56 and chromogranin were negative. Further investigation showed extensive abdominal carcinomatosis with cerebral metastasis. The patient succumbed after 1 cycle of chemotherapy with single-agent 5-fluorouracil 
(5-FU), less than 3 months after tumor debulking. 1 year and 9 months prior, the patient was diagnosed with acute deep venous thrombosis in the right popliteal and peroneal veins which was believed to be the result of a very sedentary lifestyle. Patient was started on warfarin. There was no family history of clotting abnormalities and outpatient hereditary and acquired hypercoagulable workup testing was negative. Half a year later, she started having diffuse abdominal pain that was more pronounced in the days preceding the onset of menstruation and tended to improve once bleeding stopped. Upper and lower endoscopies were performed, which showed gastritis and mild diffuse diverticulosis in the sigmoid and ascending colon, respectively. Another 6 months later, the patient came in with vomiting and severe abdominal pain, more prominent in the right upper quadrant. On abdominal exam, Murphy's sign was positive. After being taken off anticoagulation, she underwent elective laparoscopic cholecystectomy, with only a few weeks of symptomatic improvement. Shortly after, rapid deterioration led to the previously detailed admission.

\section{Discussion}

The incidence of SB cancer in the U.S. has almost doubled between 1973 and 2004 (2). The number of African Americans affected is about twice that of Caucasians, with most patients being diagnosed in the 5 to 7 th decade of life (5). Early diagnosis poses a challenge, as presenting symptoms, are often-times vague and difficult to recognize, which leads to an average delay in diagnosis of $6-12$ months $(3,4)$. The typical presentation includes, as seen in our patient, insidious and nonspecific abdominal discomfort, vomiting, nausea, weight loss and anemia (6). Tumors are generally diagnosed in advanced stages, when patients come in, as our patient did, with an acute GI emergency (usually SB obstruction (40\%) or GI bleed $(24 \%)(3,4)$.

The four major types of SB malignancies are neuroendocrine tumors $(37.4 \%)$, adenocarcinomas $(36.9 \%)$, lymphomas $(17.3 \%)$ and stromal tumors $(8.4 \%)(2)$. Their incidence has increased in recent decades, a trend that shadows that of large bowel tumors (5). In a retrospective study on 2123 patients with small-bowel adenocarcinoma, the 5-year survival was $34.9 \%$, lower than that of colon cancer (51.5\%) (7). Large and SB cancers are geographically correlated and patients likely share common environmental risk factors, such as alcohol and smoking $(1,5)$. Increased consumption of refined carbohydrates, red meat and smoked foods have also been linked to the development of SB malignancies (8). The segment most commonly involved in SB adenocarcinomas is the duodenum, followed by the jejunum (9). However, specific location within the small intestine does not appear to play a role as far as prognosis for SB adenocarcinoma is concerned (6). SB neuroendocrine tumors, on the other hand, are uncommonly found in the jejunum $(<8 \%)(10)$. While proximal tumors are usually nonfunctional, distal malignancies can be serotonin-producing tumors that present with diarrhea, flushing, carcinoid heart disease, bronchial constriction and abdominal pain, as seen in our patient (10).

GI tract adenocarcinomas with NED are very rare. To our knowledge, they were previously reported in the esophagus, duodenum, stomach, ileum, colon and rectum but not in the jejunum (11-15). Adenocarcinomas with NED are not to

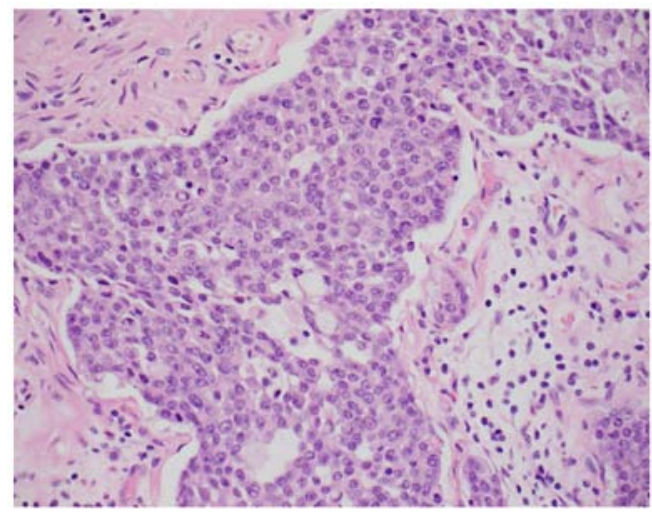

Figure 1. Infiltrating adenocarcinoma with areas overexpressing synaptophysin, consistent with neuroendocrine differentiation. Hematoxylin and eosin staining; magnification, $\mathrm{x} 400$.

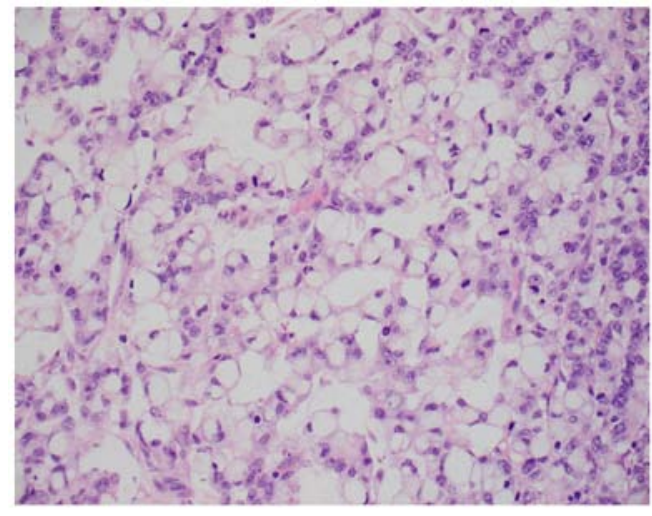

Figure 2. Infiltrating adenocarcinoma with solid growth pattern and Signet-ring cells. Hematoxylin and eosin staining; magnification, x400.

be confused with mixed adenoneuroendocrine carcinomas (MANEC). MANEC are also very rare malignancies with both gland-forming epithelial and neuroendocrine components (16). For a tumor to be classified as MANEC, however, both the adenocarcinoma and the neuroendocrine component must be identified in a proportion of at least $30 \%$ (16). A case of adenocarcinoma with patchy neuroendocrine cells accounting for less than $30 \%$, such as in this case, will not be classified as MANEC. To our knowledge, no reports of MANEC tumors in the jejunum have been published to date either.

Older imaging studies have proven limited in their evaluation of the SB: Barium small bowel follow-through has a modest sensitivity (60\%) in tumor detection and so do CT and magnetic resonance imaging (MRI) (47-80\%), whereas push enteroscopy can usually assess only up to $40 \%$ of the length of the SB (17). With the addition of CT enterography/ enteroclysis, designed specifically to evaluate the small intestine, accuracy for SB neoplasm identification has increased to $84.7-92.7 \%(18,19)$. Magnetic resonance enterography has similar diagnostic performance but is generally more expensive and less available (20).

Capsule endoscopy, although time consuming, is a good imaging option when standard bowel evaluation does not reveal obvious pathology, yet clinical suspicion remains high. In a meta-analysis on 24 studies (530 patients, each of whom had previously undergone a mean of 6.77 diagnostic studies, with 
negative results), capsule endoscopy identified $87 \%$ of tumors while other techniques (push enteroscopy, small bowel series, or colonoscopy with ileoscopy) identified 13\% (21). However, if a SB tumor is present, retention of the capsule can occur in up to $10 \%$ of patients, which subjects them to more invasive methods (enteroscopy or surgery) for capsule retrieval $(22,23)$.

Overtube-assisted enteroscopy is specifically useful in the diagnosis of SB tumors because it can visualize the entire small intestine, has a low rate of complications and allows for specimen biopsy (23). Overtube-assisted enteroscopy includes double-balloon, single-balloon and spiral enteroscopy (23). While the concordance rate between overtube-assisted enteroscopy and capsule endoscopy appears higher than $90 \%$, instances were reported in which one study was superior to the other in identifying lesions, and vice versa (23). Thus, when clinical suspicion for a SB malignancy remains high, both techniques can be utilized, for improved diagnostic accuracy (23). A major limiting factor, however, is that capsule endoscopy and overtube-assisted enteroscopy are not yet widely available.

As of yet, there is no standard treatment for SB cancer, includingnone foradenocarcinomawithNED.Recommendations state that mixed tumors with well-differentiated endocrine cells should be treated as adenocarcinomas, whereas, if the endocrine cells are poorly differentiated, the mass ought to be treated as a poorly differentiated endocrine carcinoma (24). While surgical resection is the treatment of choice for localized SB tumors, no specific recommendations have been formulated for unresectable or disseminated disease (4). In metastatic disease, surgery at the primary tumor site is often times needed because of obstruction or bleeding (4). Despite increased use of surgery and adjuvant chemotherapy (8.1\% in 1985 vs. $23.8 \%$ in 2005), survival rates in SB cancer have not improved (2). The chemotherapy of choice for adjuvant treatment in metastatic small bowel adenocarcinoma has been single-agent 5-fluorouracil (5-FU) (17). Adding a platinum agent to 5-FU might be beneficial (17). CAPOX (combination of capecitabine and oxaliplatin), FOLFOX (folinic acid/5-FU/oxaliplatin) and FOLFIRI (folinic acid/5-FU/irinotecan) are promising therapeutic options (17). More clinical trials are needed to assess treatment methods for this rare malignancy.

To our knowledge, a case of adenocarcinomas with NED has never been reported in the jejunum. Although SB cancer makes up a relatively small percentage of all cancer types, its incidence has dramatically risen in the last 40 years. However, a more active surgical and chemotherapeutic approach, has not resulted in improved survival rates. When patients present with insidious and nonspecific abdominal discomfort, a possible SB malignancy can be considered. If common diagnostic techniques are negative but clinical suspicion remains high, investigative studies can be expanded to include newer imaging. This could potentially identify these tumors earlier, prevent the development of emergent GI complications and offer a chance for cure. More clinical trials are needed in order to find new and improved methods for diagnosis and treatment.

\section{Acknowledgements}

The authors would like to thank Dr Dennis Bloomfield (Director of Clinical Research at Richmond University Medical Center, New York, NY, USA) for his editorial assistance.

\section{Funding}

No funding was received.

\section{Availability of data and materials}

All data generated or analyzed during this study are included in this published article.

\section{Authors' contributions}

EC, WZ and SS followed-up the patient. EC, WZ, IC and AP conceived and designed the case report. EC, IC and AP performed the literature review. EC, WZ, IC, AP and SS wrote the manuscript. All authors revised the manuscript critically. All authors have read and approved the final draft of the manuscript.

\section{Ethics approval and consent to participate}

Not applicable.

\section{Patient consent for publication}

The patient provided written informed consent for the publication of this case report and any associated images.

\section{Competing interests}

The authors declare that they have no competing interests.

\section{References}

1. Schottenfeld D, Beebe-Dimmer JL and Vigneau FD: The epidemiology and pathogenesis of neoplasia in the small intestine. Ann Epidemiol 19: 58-69, 2009.

2. Bilimoria KY, Bentrem DJ, Wayne JD, Ko CY, Bennett CL and Talamonti MS: Small bowel cancer in the United States: Changes in epidemiology, treatment, and survival over the last 20 years. Ann Surg 249: 63-71, 2009.

3. Dabaja BS, Suki D, Pro B, Bonnen M and Ajani J: Adenocarcinoma of the small bowel: Presentation, prognostic factors, and outcome of 217 patients. Cancer 101: 518-526, 2004.

4. Li J, Wang Z, Liu N, Hao J and Xu X: Small bowel adenocarcinoma of the jejunum: A case report and literature review. World J Surg Oncol 14: 177, 2016.

5. Haselkorn T, Whittemore AS and Lilienfeld DE: Incidence of small bowel cancer in the United States and worldwide: Geographic, temporal, and racial differences. Cancer Causes Control 16: 781-787, 2005.

6. Chaiyasate K, Jain AK, Cheung LY, Jacobs MJ and Mittal VK: Prognostic factors in primary adenocarcinoma of the small intestine: 13-year single institution experience. World J Surg Oncol 6: 12, 2008

7. Young JI, Mongoue-Tchokote S, Wieghard N, Mori M, Vaccaro GM, Sheppard BC and Tsikitis VL: Treatment and survival of small-bowel adenocarcinoma in the United States: A comparison with colon cancer. Dis Colon Rectum 59: 306-315, 2016.

8. Aparicio T, Zaanan A, Svrcek M, Laurent-Puig P, Carrere N, Manfredi S, Locher C and Afchain P: Small bowel adenocarcinoma: Epidemiology, risk factors, diagnosis and treatment. Dig Liver Dis 46: 97-104, 2014.

9. Sun KK, Wu X, Liu G, Qian H and Shen X: Primary adenocarcinoma of the small intestine presenting as superior mesenteric artery syndrome: A case report. Oncol Lett 11: 1903-1906, 2016.

10. Scherübl H, Jensen RT, Cadiot G, Stölzel U and Klöppel G: Neuroendocrine tumors of the small bowels are on the rise: Early aspects and management. World $\mathbf{J}$ Gastrointest Endosc 2: 325-334, 2010. 
11. Wang KL, Yang Q, Cleary KR, Swisher SG, Correa AM, Komaki R, Ajani JA, Rashid A, Hamilton SR and Wu TT: The significance of neuroendocrine differentiation in adenocarcinoma of the esophagus and esophagogastric junction after preoperative chemoradiation. Cancer 107: 1467-1474, 2006.

12. Fresno MF, Floriano P, Díaz Iglesias JM, Ablanedo P, Pérez del Río MJ, Barneo L and Herrero A: Neuroendocrine carcinoma of the ampullar region with oat-cell histological features and adenocarcinoma. Rev Esp Enferm Dig 89: 60-64, 1997 (In Spanish)

13. Kim JJ, Kim JY, Hur H, Cho YK and Han SU: Clinicopathologic significance of gastric adenocarcinoma with neuroendocrine features. J Gastric Cancer 11: 195-199, 2011.

14. Matulewicz RS, Fryer JP, Yang XJ, Goyal R and Hairston JC: Renal transplantation in the setting of prior urinary diversion: A case of poorly differentiated adenocarcinoma in an ileal conduit. Urol Case Rep 3: 53-55, 2015.

15. Zeng YJ, Lai W, Liu L, Wu H, Luo XX, Wang J and Chu ZH: Prognostic significance of neuroendocrine differentiation in colorectal adenocarcinoma after radical operation: A meta-analysis. J Gastrointest Surg 18: 968-976, 2014.

16. Rindi G, Arnold R, Bosman FT, Capella C, Klimstra DS Klöppel G, Komminoth P and Solcia E: Nomenclature and classification of neuroendocrine neoplasms of the digestive system. In: WHO classification of tumours of the digestive system, 4th edition. Bosman FT, Carneiro F, Hruban RH and Theise ND (eds). International agency for research on cancer (IARC), Lyon, pp13-14, 2010.

17. Overman MJ: Recent advances in the management of adenocarcinoma of the small intestine. Gastrointest Cancer Res 3: 90-96, 2009.

18. Hakim FA, Alexander JA, Huprich JE, Grover M and Enders FT: CT-enterography may identify small bowel tumors not detected by capsule endoscopy: Eight years experience at Mayo Clinic Rochester. Dig Dis Sci 56: 2914-2919, 2011.
19. Pilleul F, Penigaud M, Milot L, Saurin JC, Chayvialle JA and Valette PJ: Possible small-bowel neoplasms: Contrast-enhanced and water-enhanced multidetector CT enteroclysis. Radiology 241: 796-801, 2006.

20. Ilangovan R, Burling D, George A, Gupta A, Marshall M and Taylor SA: CT enterography: Review of technique and practical tips. Br J Radiol 85: 876-886, 2012.

21. Lewis BS, Eisen GM and Friedman S: A pooled analysis to evaluate results of capsule endoscopy trials. Endoscopy 37: 960-965, 2005.

22. Rondonotti E, Pennazio M, Toth E, Menchen P, Riccioni ME, De Palma GD, Scotto F, De Looze D, Pachofsky T, Tacheci I, et al: Small-bowel neoplasms in patients undergoing video capsule endoscopy: A multicenter European study. Endoscopy 40: 488-495, 2008

23. Sulbaran M, de Moura E, Bernardo W, Morais C, Oliveira J, Bustamante-Lopez L, Sakai P, Mönkemüller $\mathrm{K}$ and Safatle-Ribeiro A: Overtube-assisted enteroscopy and capsule endoscopy for the diagnosis of small-bowel polyps and tumors: A systematic review and meta-analysis. Endosc Int Open 4: E151-E163, 2016.

24. Hervieu V and Scoazec JY: Mixed endocrine tumors. Ann Pathol 25: 511-528, 2005 (In French).

(i) $(9$ This work is licensed under a Creative Commons Attribution-NonCommercial-NoDerivatives 4.0 International (CC BY-NC-ND 4.0) License. 